\title{
Magnetohydrodynamic modes in a periodic magnetic steady state medium
}

\author{
A. Marcu ${ }^{1}$, I. Ballai ${ }^{2}$, and B. Pintér ${ }^{2}$ \\ ${ }^{1}$ Department of Theoretical and Computational Physics, Babes-Bolyai University, 1, M. Kogalniceanu, 400084 Cluj-Napoca, Romania \\ e-mail: amarc@phys.ubbcluj.ro \\ 2 Solar Physics \& upper-Atmosphere Research Group (SPARG), Department of Applied Mathematics, University of Sheffield, Hicks Building, \\ Hounsfield Road, Sheffield, S3 7RH, UK
}

Received 26 August 2005 / Accepted 8 December 2005

ABSTRACT

The spatial structuring of solar and space plasmas is known to have a dispersive effect on waves. Many solar features possess a periodic structure with structures having alternating properties. Here the effect of periodic alternation of magnetic slabs on wave propagation is studied when the equilibrium has a steady motion (in line with observations). The dispersion relation for linear compressional waves is derived and analysed. The propagation of waves is studied, in particular modelling the cases of propagation in penumbral filamentary structures in the photosphere, the plume/interplume region and spaghetti structures in the solar wind. Depending on the width of structures (or the wavelength of oscillations) waves have different behaviour. It is shown that the strength of the equilibrium flow has a strong influence on the propagation speed and character of the wave.

Key words. Sun: oscillations - Sun: magnetic fields

\section{Introduction}

Recent observational evidence for the existence of waves and oscillations in the solar atmosphere has invigorated theoretical developments in solar plasma physics. Waves are responsible for carrying energy and momentum, creating instabilities, generating phenomena like magnetic reconnection, resonant absorption, phase mixing, etc. They can also serve as unique tools for plasma diagnostics due to their ability to carry information about the medium in which they propagate.

One of the basic features of solar and space plasmas is that these media are always dynamic, showing steady flows on all time and space scales; this property is confirmed by recent ground and space-born satellite observations. Steady flows have been observed in the photosphere (Krijger et al. 2002; Bellot Robio et al. 2003), chromosphere (Athay \& Dere 1991), corona (Winebarger et al. 2002; Gabriel et al. 2003) and beyond, in the solar wind (Buchlin \& Hassler 2000; Gabriel et al. 2003). Therefore, all theoretical models should include the presence of an equilibrium steady flow. Equilibrium flows are known to introduce a series of new effects such as Kelvin-Helmholtz instabilities, negative energy waves, resonant instabilities, enhancement or reduction of wave absorption in plasmatic structures, etc.

Observations have revealed that the magnetic field is not distributed smoothly over the solar surface but is structured; the magnetic field tends to accumulate in magnetic flux tubes or loops. In the photosphere, the magnetic field is concentrated in thin flux tubes with field strengths exceeding $1000 \mathrm{G}$. These flux tubes extend well into the chromosphere and corona, contributing to the net heating of the solar upper layers, creating the solar spiculae (Roberts 1979; James \& Erdélyi 2002; De Pontieu et al. 2004) and even contributing to acceleration of the solar wind. The solar wind is also very structured with discontinuities (rotational and tangential) appearing with a frequency of $1 \mathrm{~h}^{-1}$ at $1 \mathrm{AU}$ (Mariani et al. 1983). These structures and discontinuities are excellent guides for magnetohydrodynamic (MHD) waves. The propagation of waves and oscillations in solar magnetic structures and at discontinuities has been studied intensively in the last few decades (Roberts 1981; Gordon \& Hollweg 1983; Edwin \& Roberts 1982, 1983; Nakariakov \& Roberts 1995; Homem et al. 2003; Ballai \& Marcu 2004; Carter 2005).

Many solar features (e.g. the granular pattern in the photosphere, the penumbra, plume/interplume region, etc.) show a transversal periodicity (or quasi-periodicity). A wave propagating along magnetic structures with a transversal periodicity is likely to "feel" the effect of this structuring provided its transversal wavelength is of the same order as the transversal spatial organisation scale. Effects of periodicity, although not perfect on the Sun's surface, could be observationally detected on a $\omega$-k diagnostic diagram.

The possibility of wave propagation in periodic structures has been discussed by Hollweg (1982), Berton \& Heyvaerts (1987, BH87, thereafter) and Uralov (2003) in the linear regime. Recently, Díaz \& Roberts (2006) studied the 
propagation of fast MHD oscillations in periodic structures, modelling prominence fibrils. The steepening of linear waves into nonlinear waves (solitons) when dispersion resulted due to periodic structures was studied by Hollweg \& Roberts (1984) and Ruderman et al. (2001).

The present paper aims to investigate the linear compressional wave propagation in a magnetically periodic structure, when weak field regions alternate with strong field regions. The dispersion relation of linear compressional waves is derived and analyzed analytically and numerically. The paper is organized as follows: in Sect. 2 we derive the dispersion relation for compressional fast and slow magnetoacoustic modes propagating in periodic structures when an equilibrium steady flow is present in the structure. Section 3 is devoted to the study of the special case of incompressible plasma. The dispersion relations in compressible plasma are derived in Sect. 4 and we apply the theoretical results to study the propagation of waves in various solar structures. In Sect. 5 we summarize and discuss our results.

\section{Derivation of dispersion relations}

We consider an ideal, perfectly conducting fluid permeated by a magnetic field of constant direction along the $z$-axis, and periodic along the $x$-axis. We suppose that wavelengths are smaller than the gravitational scale-height, i.e. the gravitational effects are neglected.

A field-aligned equilibrium steady flow is present in the system. We suppose that the medium consists of alternating magnetic slabs (with widths $L_{\mathrm{i}}$ and $L_{\mathrm{e}}$ ) with a homogeneous magnetic field inside them $\left(B_{\mathrm{i}}\right.$ and $\left.B_{\mathrm{e}}\right)$ and a sharp discontinuity at the boundary as shown in Fig. 1. Let us denote the periodicity of the medium and $\rho_{\mathrm{i}}$ and $\rho_{\mathrm{e}}$ the (homogeneous) densities in the two regions by $L=L_{\mathrm{i}}+L_{\mathrm{e}}$. The continuity of the total pressure at each boundary requires an equation of the type

$\frac{\mathrm{d}}{\mathrm{d} x}\left(p_{0}+\frac{B_{0}^{2}}{2 \mu}\right)=0$,

which, in particular, leads to a density contrast in two adjacent regions described by

$R_{\rho}=\frac{\rho_{\mathrm{i}}}{\rho_{\mathrm{e}}}=\frac{2 c_{0 \mathrm{e}}^{2}+\gamma c_{\mathrm{Ae}}^{2}}{2 c_{0 \mathrm{i}}^{2}+\gamma c_{\mathrm{Ai}}^{2}}$,

where $c_{0 \mathrm{i}, \mathrm{e}}$ and $c_{\mathrm{Ai}, \mathrm{e}}$ denote the sound and Alfvén speeds in the two layers, $\gamma$ is the adiabatic index and $\mu$ is the magnetic permeability.

We perturb the system and write all physical quantities in the form $f_{0}+f$, where $f_{0}$ are the equilibrium values and $f$ their Eulerian perturbations. We suppose small but finite deviation from the equilibrium value, i.e. we concentrate on linear waves only. In both regions the plasma dynamics is described by the system of linearized ideal MHD equations

$$
\begin{aligned}
& \frac{\partial \rho}{\partial t}+\boldsymbol{v} \cdot \nabla \rho_{0}+\boldsymbol{v}_{\mathbf{0}} \cdot \nabla \rho=-\rho_{0} \nabla \cdot \boldsymbol{v} \\
& \frac{\partial \boldsymbol{v}}{\partial t}+v_{0} \frac{\partial \boldsymbol{v}}{\partial z}=-\frac{\nabla p}{\rho_{0}}+\frac{1}{\mu \rho_{0}}(\nabla \times \boldsymbol{b}) \times \boldsymbol{B}_{0},
\end{aligned}
$$

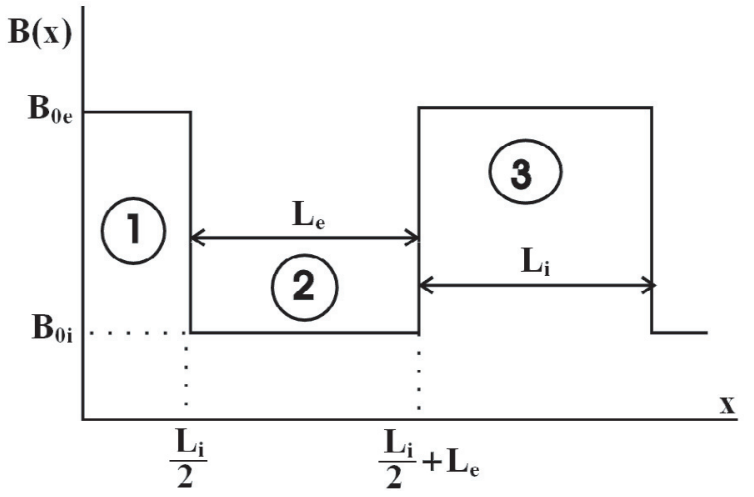

Fig. 1. Profile of the magnetic field in a periodic equilibrium state.

$\frac{\partial \boldsymbol{b}}{\partial t}=\nabla \times\left(\boldsymbol{v}_{\mathbf{0}} \times \boldsymbol{b}\right)-\nabla \times\left(\boldsymbol{B}_{\mathbf{0}} \times \boldsymbol{v}\right)$,
$\frac{\partial p}{\partial t}+\boldsymbol{v} \cdot \nabla p_{0}+v_{0} \frac{\mathrm{d} p}{\mathrm{~d} z}=-\rho_{0} c_{0}^{2} \nabla \cdot \boldsymbol{v}$.

Here, $p$ and $\rho$ are the kinetic pressure and density. The perturbations of the magnetic field and velocity are denoted by $\boldsymbol{b}$ and $\boldsymbol{v}$. Since the equilibrium quantities depend on $x$ only, we write the perturbations as $f=\hat{f}(x) \mathrm{e}^{\mathrm{i}\left(\omega t-k_{y} y-k_{z} z\right)}$. Assuming $\hat{v}_{y}=\hat{b}_{y}=0$, the linearized ideal MHD equations can be cast into a single equation for the $x$-component of the velocity perturbation describing compressional waves (Roberts 1981)

$\frac{\mathrm{d}^{2} \hat{v}_{x}}{\mathrm{~d} x^{2}}-\left(q^{2}+k_{y}^{2}\right) \hat{v}_{x}=0$,

where $\Omega=\omega-k_{z} v_{0}$ is the Doppler shifted frequency and the magnetoacoustic parameter, $q(x)$, is defined as

$q(x)^{2}=\frac{\left(k_{z}^{2} c_{\mathrm{A}}^{2}-\Omega^{2}\right)\left(k_{z}^{2} c_{0}^{2}-\Omega^{2}\right)}{\left(c_{0}^{2}+c_{\mathrm{A}}^{2}\right)\left(k_{z}^{2} c_{\mathrm{T}}^{2}-\Omega^{2}\right)}$,

with $c_{\mathrm{T}}=c_{0} c_{\mathrm{A}} /\left(c_{0}^{2}+c_{\mathrm{A}}^{2}\right)^{1 / 2}$ being the tube (cusp) velocity. The quantity $q(x)^{2}$ is constant in each region and it can take both positive and negative values with $\omega^{2}$ and $k^{2}$ real.

The solutions of Eq. (7) inside and outside the slab can be written as

$\left\{\begin{array}{l}\hat{v}_{x \mathrm{i}}=\alpha_{\mathrm{i}} \mathrm{e}^{q_{\mathrm{i}}^{\prime} x}+\beta_{\mathrm{i}} \mathrm{e}^{-q_{\mathrm{i}}^{\prime} x}, \\ \hat{v}_{x \mathrm{e}}=\alpha_{\mathrm{e}} \mathrm{e}^{q_{\mathrm{e}}^{\prime} x}+\beta_{\mathrm{e}} \mathrm{e}^{-q_{\mathrm{e}}^{\prime} x},\end{array}\right.$

where $q_{\mathrm{i}}^{\prime}=\sqrt{q_{\mathrm{i}}^{2}+k_{y}^{2}}, q_{\mathrm{e}}^{\prime}=\sqrt{q_{\mathrm{e}}^{2}+k_{y}^{2}}$ and the coefficients $\alpha_{\mathrm{i}}$, $\beta_{\mathrm{i}}, \alpha_{\mathrm{e}}$ and $\beta_{\mathrm{e}}$ are four constants which can be determined by using the matching conditions at the boundaries of two adjacent regions (at $x=L_{\mathrm{i}} / 2$ and $x=L_{\mathrm{e}}+L_{\mathrm{i}} / 2$ ). The matching conditions require the continuity of the normal component of velocity and total pressure

$\left.\frac{\hat{v}_{x \mathrm{i}}}{\hat{v}_{x \mathrm{e}}}\right|_{x=\frac{L_{\mathrm{i}}}{2}}=A=\left.\frac{\hat{v}_{x \mathrm{i}}}{\hat{v}_{x \mathrm{e}}}\right|_{x=\frac{L_{\mathrm{i}}}{2}+L_{\mathrm{e}}}$,

$\left.S \frac{\mathrm{d} \hat{v}_{x \mathrm{i}}}{\mathrm{d} x}\right|_{x=\frac{L_{\mathrm{i}}}{2}}=\left.A \frac{\mathrm{d} \hat{v}_{x \mathrm{e}}}{\mathrm{d} x}\right|_{x=\frac{L_{\mathrm{i}}}{2}}$,

$\left.S \frac{\mathrm{d} \hat{v}_{x \mathrm{i}}}{\mathrm{d} x}\right|_{x=\frac{L_{\mathrm{i}}}{2}+L_{\mathrm{e}}}=\left.A \frac{\mathrm{d} \hat{v}_{x \mathrm{e}}}{\mathrm{d} x}\right|_{x=\frac{L_{\mathrm{i}}}{2}+L_{\mathrm{e}}}$, 
where

$A=\frac{\omega-k_{z} v_{0 \mathrm{i}}}{\omega-k_{z} v_{0 \mathrm{e}}}$

$S=\frac{\rho_{\mathrm{i}}}{\rho_{\mathrm{e}}} \frac{k_{z}^{2} c_{\mathrm{Ai}}^{2}-\Omega_{\mathrm{i}}^{2}}{k_{z}^{2} c_{\mathrm{Ae}}^{2}-\Omega_{\mathrm{e}}^{2}} \frac{\sqrt{q_{\mathrm{e}}^{2}+k_{y}^{2}}}{\sqrt{q_{\mathrm{i}}^{2}+k_{y}^{2}}}=\frac{\rho_{\mathrm{i}}}{\rho_{\mathrm{e}}} \frac{L_{\mathrm{i}}}{L_{\mathrm{e}}} \frac{\theta_{\mathrm{e}}}{\theta_{\mathrm{i}}} \frac{k_{z}^{2} c_{\mathrm{Ai}}^{2}-\Omega_{\mathrm{i}}^{2}}{k_{z}^{2} c_{\mathrm{Ae}}^{2}-\Omega_{\mathrm{e}}^{2}}$,

with

$\theta_{\mathrm{i}}=L_{\mathrm{i}} \sqrt{q_{\mathrm{i}}^{2}+k_{y}^{2}} ; \quad \theta_{\mathrm{e}}=L_{\mathrm{e}} \sqrt{q_{\mathrm{e}}^{2}+k_{y}^{2}}$.

For simplicity we consider the case $k_{y}=0$. Solving simultaneously the system of Eqs. (10), (11) we obtain the dispersion relation for linear compressional waves propagating in periodic structures.

Due to the periodicity of the medium we employ a method widely used in solid state physics. We introduce the quantity $K_{0}$ (called the Bloch wavenumber) which here plays the same role as the wavenumber $k_{x}$ in a homogeneous medium. According to Bloch's theorem, a solution of Eq. (7) which is bounded at infinity can be written as

$\hat{v}_{x}=a F(x) \exp \left[\mathrm{i} K_{0} x\right]+b F(-x) \exp \left[-\mathrm{i} K_{0} x\right]$,

where $F(x)$ is a periodic function with the period $L$. Then

$\frac{\hat{v}(L)}{\hat{v}(0)}=\cos K_{0} L$.

In the case of surface $\left(q_{\mathrm{i}}^{2}>0\right)$ and body $\left(q_{\mathrm{i}}^{2}<0\right)$ modes, the dispersion relations can be written as

$\frac{\cos \left(K_{0} L\right)}{\sinh \left(\theta_{\mathrm{i}}\right) \sinh \left(\theta_{\mathrm{e}}\right)}=\operatorname{coth}\left(\theta_{\mathrm{i}}\right) \operatorname{coth}\left(\theta_{\mathrm{e}}\right)+\frac{1}{2}\left(S+\frac{1}{S}\right)$,

$\frac{\cos \left(K_{0} L\right)}{\sin \left(\theta_{\mathrm{i}}\right) \sinh \left(\theta_{\mathrm{e}}\right)}=\cot \left(\theta_{\mathrm{i}}\right) \operatorname{coth}\left(\theta_{\mathrm{e}}\right)+\frac{1}{2}\left(S-\frac{1}{S}\right)$.

The dispersion curves $\omega\left(k_{z}\right)$ will depend upon the Bloch's wavenumber, $K_{0}$, as a parameter. Although these equations are similar to the equations derived by BH87, the quantities that appear in these equations contain information about the equilibrium flow.

According to the relative magnitude of the characteristic speeds $c_{0}, c_{\mathrm{A}}$, and $c_{\mathrm{T}}$ in the internal and external regions, various situations are possible depending on the signs of the quantities $q_{\mathrm{i}}^{2}$ and $q_{\mathrm{e}}^{2}$.

\section{Incompressible modes}

A first insight into the properties of the possible modes propagating in periodic steady plasma structures can be obtained by considering the incompressible plasma limit which allows for a relatively simple analytical solution and a better understanding of the behaviour of the different modes with respect to the equilibrium steady flow and periodicity. To obtain the incompressible plasma approximation, we take $c_{0}, c_{\mathrm{e}} \rightarrow \infty(\gamma \rightarrow \infty)$. In this case, from Eq. (8) we obtain $q^{2}(x)=\left|k_{z}\right|>0$, i.e. only surface non-leaky (trapped) modes are allowed to propagate. We introduce the dimensionless arguments

$\theta=k_{z} L_{\mathrm{i}}, \quad \Phi=K_{0} L$.

Using these notations, the dispersion relation of surface waves becomes

$\frac{\cos \Phi}{\sinh (\theta) \sinh \left(\frac{\theta}{R_{L}}\right)}-\operatorname{coth}(\theta) \operatorname{coth}\left(\frac{\theta}{R_{L}}\right)=\frac{1}{2}\left(S+\frac{1}{S}\right)$,

where

$S=R_{\rho} \frac{c_{\mathrm{Ai}}^{2}-\left(c-v_{0 \mathrm{i}}\right)^{2}}{c_{\mathrm{Ae}}^{2}-\left(c-v_{0 \mathrm{e}}\right)^{2}}, \quad R_{L}=\frac{L_{\mathrm{i}}}{L_{\mathrm{e}}}$,

and $c=\omega / k_{z}$ is the phase speed of the waves. The numerical investigation of these modes requires representing the function $\mathrm{c}(\theta)$ satisfying Eqs. (17) and (18), with $\Phi$ as a parameter. Inverting Eq. (17) yields

$$
\begin{aligned}
& S^{2}-2 \frac{\cos \Phi-\cosh (\theta) \cosh \left(\frac{\theta}{R_{L}}\right)}{\sinh (\theta) \sinh \left(\frac{\theta}{R_{L}}\right)} S+1= \\
& S^{2}-2 U S+1=0,
\end{aligned}
$$

with

$\frac{\cos \Phi-\cosh (\theta) \cosh \left(\frac{\theta}{R_{L}}\right)}{\sinh (\theta) \sinh \left(\frac{\theta}{R_{L}}\right)}=U$.

Equation (19) admits two roots given by

$S^{( \pm)}=U \pm \sqrt{U^{2}-1}$

where $S^{(+)}$and $S^{(-)}$correspond to sausage and kink modes, respectively. In Eq. (21) we have to impose that $U^{2} \geq 1$, i.e. the quantity $S^{( \pm)}$is a real number, i.e. fast surface waves propagate in an incompressible periodically structured plasma provided

$\cosh k_{z}\left(L_{\mathrm{i}}+L_{\mathrm{e}}\right) \leq \cos K_{0} L \leq \cosh k_{z}\left(L_{\mathrm{i}}-L_{\mathrm{e}}\right)$.

The dispersion relation of fast magnetoacoustic modes propagating in periodic structures in the incompressible limit is given by the roots of the polynomial (in $c$ )

$$
\begin{array}{r}
c^{2}\left(R_{\rho}-S^{( \pm)}\right)+2 c\left(S^{( \pm)} v_{0 \mathrm{e}}-\right. \\
\left.R_{\rho} v_{0 \mathrm{i}}\right)-R_{\rho}\left(c_{\mathrm{Ai}}^{2}-v_{0 \mathrm{i}}^{2}\right) \\
+S_{ \pm}\left(c_{\mathrm{Ae}}^{2}-v_{0 \mathrm{e}}^{2}\right)=0 .
\end{array}
$$

Equation (22) possesses two real roots corresponding to two possible sausage or kink modes (propagating parallel and anti-parallel to the magnetic field) provided

$\frac{R_{\rho} S^{( \pm)}}{R_{\rho}-S^{( \pm)}}>\frac{S^{( \pm)} c_{\mathrm{Ae}}^{2}-R_{\rho} c_{\mathrm{Ai}}^{2}}{\left(v_{0 \mathrm{i}}-v_{0 \mathrm{e}}\right)^{2}}$

If $v_{0 \mathrm{i}}=v_{0 \mathrm{e}}=0$, we recover the result obtained by BH87.

Before turning to the general case, let us first discuss two particular cases, $R_{L} \rightarrow 0$ (the isolated slab) and $R_{L} \rightarrow \infty$ (homogeneous plasma). For an isolated tube, $U=-\operatorname{coth} \theta$, so 
by means of Eq. (21) the phase speed of fast surface sausage modes is

$$
\begin{aligned}
c^{(+) 2}-2 c^{(+)} & \frac{v_{0 \mathrm{e}} \tanh \frac{\theta}{2}+v_{0 \mathrm{i}} R_{\rho}}{\tanh \frac{\theta}{2}+R_{\rho}} \\
& -\frac{\left(c_{\mathrm{Ae}}^{2}-v_{0 \mathrm{e}}^{2}\right) \tanh \frac{\theta}{2}+\left(c_{\mathrm{Ai}}^{2}-v_{0 \mathrm{i}}^{2}\right) R_{\rho}}{\tanh \frac{\theta}{2}+R_{\rho}}=0,
\end{aligned}
$$

while the propagation speed of fast surface kink modes is given by the roots of

$$
\begin{aligned}
& c^{(-) 2}-2 c^{(-)} \frac{v_{0 \mathrm{e}} \operatorname{coth} \frac{\theta}{2}+v_{0 \mathrm{i}} R_{\rho}}{\operatorname{coth} \frac{\theta}{2}+R_{\rho}} \\
&-\frac{\left(c_{\mathrm{Ae}}^{2}-v_{0 \mathrm{e}}^{2}\right) \operatorname{coth} \frac{\theta}{2}+\left(c_{\mathrm{Ai}}^{2}-v_{0 \mathrm{i}}^{2}\right) R_{\rho}}{\operatorname{coth} \frac{\theta}{2}+R_{\rho}}=0,
\end{aligned}
$$

identical to the results obtained by Nakariakov \& Roberts (1995). This form of Eqs. (24) and (25) allows us to interpret the effect of the equilibrium flow. In the last two equations, the second terms owe their existence to the equilibrium internal and external flows. If these flows were set to be zero, we would recover the results obtained by $\mathrm{BH} 87$.

In the limit of homogeneous plasma, we let $R_{L} \rightarrow \infty$ and we obtain the well-known propagation speeds for sausage and kink modes

$c^{(+)}= \pm c_{\mathrm{Ai}}+v_{0 \mathrm{i}}, \quad c^{(-)}= \pm c_{\mathrm{Ae}}+v_{0 \mathrm{e}}$,

where the \pm indicates forward (parallel to the magnetic field) and backward (anti-parallel to the magnetic field) propagation.

Turning to the general case, we can derive analytical solutions for the phase speed of sausage and kink modes propagating in the incompressible periodic plasma in two extreme limits corresponding to a thin $(\theta \ll 1)$ and thick $(\theta \gg 1)$ slab. If the slab is considered thin (also known as the long wavelength limit) then, from the definition of $U$, we have for small values of $\theta$

$U \approx-\frac{2 R_{L}}{\theta^{2}} \sin ^{2} \frac{\Phi}{2}$

which for sausage and kink modes leads to the approximate expressions

$S^{(+)} \approx \frac{1}{2 U}, \quad S^{(-)} \approx 2 U$.

These expressions can now be used to obtain the phase speeds of fast sausage and kink modes as

$$
\begin{aligned}
c^{(+) 2}-2 c^{(+)} & \frac{4 \frac{R_{L} R_{\rho}}{\theta^{2}} \sin ^{2} \frac{\Phi}{2} v_{0 \mathrm{i}}+v_{0 \mathrm{e}}}{1+4 \frac{R_{L} R_{\rho}}{\theta^{2}} \sin ^{2} \frac{\Phi}{2}} \\
& -\frac{4 \frac{R_{L} R_{\rho}}{\theta^{2}} \sin ^{2} \frac{\Phi}{2}\left(c_{\mathrm{Ai}}^{2}-v_{0 \mathrm{i}}^{2}\right)+c_{\mathrm{Ae}}^{2}-v_{0 \mathrm{e}}^{2}}{1+4 \frac{R_{L} R_{\rho}}{\theta^{2}} \sin ^{2} \frac{\Phi}{2}}=0,
\end{aligned}
$$

and

$$
\begin{aligned}
c^{(-) 2}-2 c^{(-)} & \frac{4 \frac{R_{L}}{\theta^{2}} \sin ^{2} \frac{\Phi}{2} v_{0 \mathrm{e}}+v_{0 i} R_{\rho}}{R_{\rho}+4 \frac{R_{L}}{\theta^{2}} \sin ^{2} \frac{\Phi}{2}} \\
& -\frac{4 \frac{R_{L}}{\theta^{2}} \sin ^{2} \frac{\Phi}{2}\left(c_{\mathrm{Ae}}^{2}-v_{0 \mathrm{e}}^{2}\right)+R_{\rho}\left(c_{\mathrm{Ai}}^{2}-v_{0 i}^{2}\right)}{R_{\rho}+4 \frac{R_{L}}{\theta^{2}} \sin ^{2} \frac{\Phi}{2}}=0 .
\end{aligned}
$$

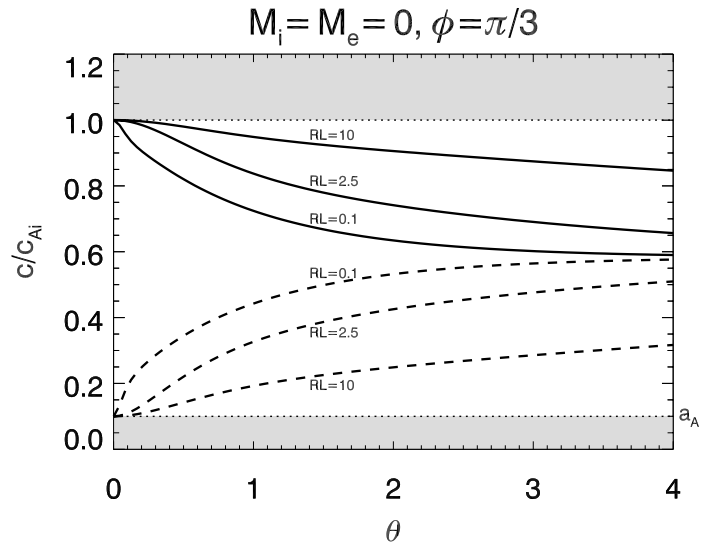

Fig. 2. The dispersion curves for sausage (solid line) and kink (dashed lines) modes as a function of the dimensionless wavelength, $\theta$, arising in an incompressible periodic plasma when no flows are present in the system. The horizontal lines correspond to $a_{\mathrm{A}}=0.1$ and $M_{\mathrm{i}}=M_{\mathrm{e}}=0$. The grey regions correspond to intervals where waves are leaky.

In the limit of a wide slab (or short wavelength limit), $S_{ \pm} \rightarrow-1$ and the dispersion relation is given by

$c_{\infty}^{2}-2 c_{\infty} \frac{v_{0 \mathrm{e}}+v_{0 i} R_{\rho}}{R_{\rho}+1}-\frac{R_{\rho}\left(c_{\mathrm{Ai}}^{2}-v_{0 \mathrm{i}}^{2}\right)+c_{\mathrm{Ae}}^{2}-v_{0 \mathrm{e}}^{2}}{R_{\rho}+1}=0$.

The possible modes propagating in an incompressible periodic structure for arbitrary $\theta$ and $R_{L}$ are shown in Figs. 2-4. All characteristic speeds are expressed in units of the internal Alfvén speeds and the following notations have been used

$a_{\mathrm{A}}=\frac{c_{\mathrm{Ae}}}{c_{\mathrm{Ai}}}, \quad M_{\mathrm{i}}=\frac{v_{0 \mathrm{i}}}{c_{\mathrm{Ai}}}, \quad M_{\mathrm{e}}=\frac{v_{0 \mathrm{e}}}{c_{\mathrm{Ai}}}$

Here we consider $a_{\mathrm{A}}=0.1, M_{\mathrm{i}}=0.04$ and $M_{\mathrm{e}}=-0.02$. Figure 2 shows the possible modes propagating in a periodic structure when no plasma flow is present in the system. Since the equilibrium state is static, modes are symmetric with respect to the horizontal axis, therefore here we only plot the forward propagating modes. The possible non-leaky modes are allowed to propagate between the two Alfvén speeds, the grey regions are the intervals where waves are leaky. The dispersion relation for linear waves in the incompressible plasma limit has been plotted for $\Phi=\pi / 3$ and for three different values of $R_{L}$ $(0.1,2.5,10)$. A simple visual inspection shows that waves are more dispersive for small values of $R_{L}$. The phase speed of sausage modes increases with increasing $R_{L}$ while the phase speed of kink modes decreases with increasing $R_{L}$. In general, sausage waves propagate faster that kink modes. For large values of $\theta$, both sausage and kink modes tend to the positive value of $c_{\infty}$ given by Eq. (31).

When an equilibrium flow is taken into account (Figs. 3, 4) the symmetry of the modes is broken although the propagation windows of the forward and backward propagating modes are shifted by an amount proportional to the flow speed. The two figures are obtained for two values of $\Phi(0, \pi / 2)$ and for three different values of $R_{L}(0.1,2.5,10)$. In fact, we could write $2 n \pi$ and $(2 n+1) \pi$ with $n$ an integer number, but for simplicity we restrict ourself only to the first term in these series. 


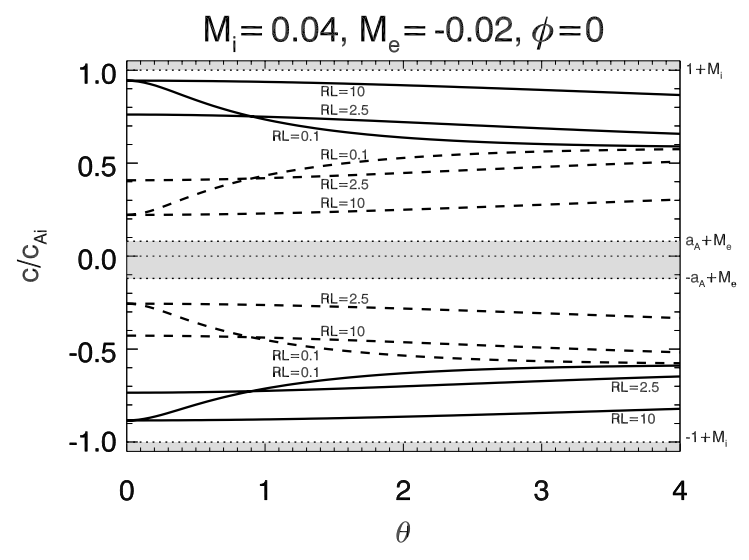

Fig. 3. The same as in Fig. 2 but now the there is an equilibrium (counter) flow of $M_{\mathrm{i}}=0.04, M_{\mathrm{e}}=-0.02$ and $\Phi=0$.

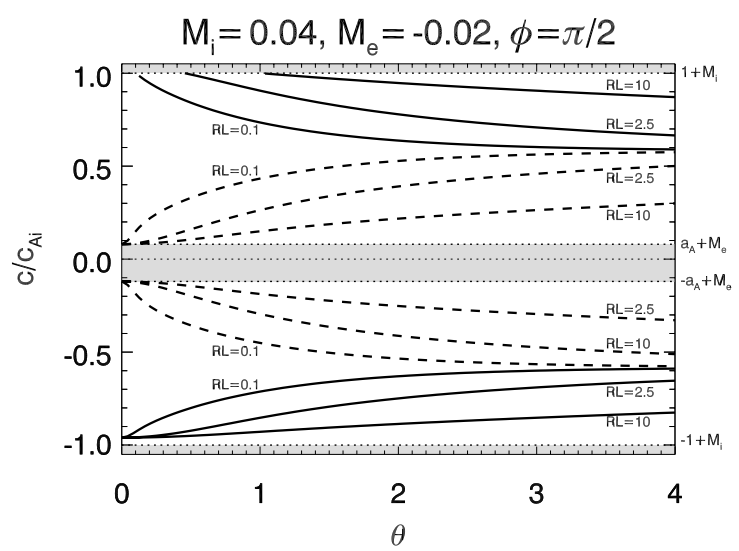

Fig. 4. The same as in Fig. 3 but for $\Phi=\pi / 2$.

When $\Phi=0$, sausage and kink modes show a significant dispersion for small values of $R_{L}$ only and a weak dispersion for $R_{L}>1$. When $\Phi \neq 0$, the behaviour of oscillating modes is similar to the case obtained in a static equilibrium. Repeating the plot for other values of $\Phi$, the dependence of the phase speed on $\theta$ is very similar to the case obtained for $\Phi=\pi / 2$; only small changes are observed for intermediate values of $\theta$.

\section{Compressible modes}

The dispersion relations for surface and body modes possess a rich variety of solutions. The full dispersion equation contains highly transcendental functions, and as a result it is impossible to obtain analytical solutions. A possible insight into the properties of linear compressional waves can be obtained by considering the two usual limiting cases: the slender or thin $(\theta \ll 1)$ and the thick-slab $(\theta \gg 1)$ approximations. Using the notations from the previous section, we define $\theta, \Phi$ and $c$ in the same way, setting

$q_{\mathrm{i}}^{2}=\frac{\left(c_{\mathrm{Ai}}^{2}-\tilde{c}_{\mathrm{i}}^{2}\right)\left(c_{\mathrm{si}}^{2}-\tilde{c}_{\mathrm{i}}^{2}\right)}{c_{\mathrm{i}}^{2}\left(c_{T \mathrm{i}}^{2}-\tilde{c}_{\mathrm{i}}^{2}\right)}, q_{\mathrm{e}}^{2}=\frac{\left(c_{\mathrm{Ae}}^{2}-\tilde{c}_{\mathrm{e}}^{2}\right)\left(c_{\mathrm{se}}^{2}-\tilde{c}_{\mathrm{e}}^{2}\right)}{c_{\mathrm{e}}^{2}\left(c_{T \mathrm{e}}^{2}-\tilde{c}_{\mathrm{e}}^{2}\right)}$,

where

$c_{\mathrm{i}}^{2}=c_{\mathrm{si}}^{2}+c_{\mathrm{Ai}}^{2}, \quad c_{\mathrm{e}}^{2}=c_{\mathrm{se}}^{2}+c_{\mathrm{Ae}}^{2}, \quad \tilde{c}_{\mathrm{i}, \mathrm{e}}=c-v_{0 \mathrm{i}, \mathrm{e}}$.
Surface modes propagate with phase speeds given by the dispersion relation

$\frac{1}{2}\left(S+\frac{1}{S}\right)=$

$\frac{\cos \Phi}{\sinh \left(q_{\mathrm{i}} \theta\right) \sinh \left(\frac{q_{\mathrm{e}} \theta}{R_{L}}\right)}-\operatorname{coth}\left(q_{\mathrm{i}} \theta\right) \operatorname{coth}\left(\frac{q_{\mathrm{e}} \theta}{R_{L}}\right)$,

where the function $S$ is defined by Eq. (12) $\left(k_{y}=0\right)$. This equation is re-arranged as

$S^{2}-S Q_{1}+1=0$,

with the roots given by

$S^{( \pm)}=\frac{Q_{1}}{2} \pm \sqrt{\frac{Q_{1}^{2}}{4}-1}$,

where the quantity $Q_{1}$ is defined as

$Q_{1}=\frac{2 \cos \Phi-2 \cosh \left(q_{\mathrm{i}} \theta\right) \cosh \left(q_{\mathrm{e}} \theta / R_{L}\right)}{\sinh \left(q_{\mathrm{i}} \theta\right) \sinh \left(q_{\mathrm{e}} \theta / R_{L}\right)}$.

In Eq. (36) we could impose a condition similar to the condition (23) to ensure that sausage and kink modes exist. In Eq. (36) $S^{( \pm)}$refers to sausage and kink modes.

A similar equation is solved for body waves. Now, the dispersion relation of sausage/kink modes is given by

$S^{( \pm)}=\frac{Q_{2}}{2} \pm \sqrt{\frac{Q_{2}^{2}}{4}+1}$,

where

$Q_{2}=\frac{2 \cos \Phi-2 \cos \left(q_{\mathrm{i}} \theta\right) \cosh \left(q_{\mathrm{e}} \theta / R_{L}\right)}{\sin \left(q_{\mathrm{i}} \theta\right) \sinh \left(q_{\mathrm{e}} \theta / R_{L}\right)}$.

In the thin slab approximation $(\theta \ll 1)$ Eq. (34) reduces to

$-\frac{4 \sin ^{2} \frac{\Phi}{2}}{\theta^{2}} \approx q_{\mathrm{i}}^{2}+\frac{q_{\mathrm{e}}^{2}}{R_{L}^{2}}+\left(S+\frac{1}{S}\right) \frac{q_{\mathrm{i}} q_{\mathrm{e}}}{R_{L}}$.

Using the definition of the $S$ functions, Eq. (40) becomes

$$
\begin{aligned}
-\frac{4 \sin ^{2} \frac{\Phi}{2}}{\theta^{2}} \approx & q_{\mathrm{i}}^{2}\left\{1+\frac{1}{R_{\rho} R_{L}} \frac{c_{\mathrm{Ae}}^{2}-\tilde{c}_{\mathrm{e}}^{2}}{c_{\mathrm{Ai}}^{2}-\tilde{c}_{\mathrm{i}}^{2}}\right\} \\
& +\frac{q_{\mathrm{e}}^{2}}{R_{L}^{2}}\left\{1+R_{\rho} R_{L} \frac{c_{\mathrm{Ai}}^{2}-\tilde{c}_{\mathrm{i}}^{2}}{c_{\mathrm{Ae}}^{2}-\tilde{c}_{\mathrm{e}}^{2}}\right\} .
\end{aligned}
$$

If $\Phi \neq 0$, the two possible modes propagate with phase speeds

$$
\begin{aligned}
c \approx & v_{0 \mathrm{i}} \pm c_{T \mathrm{i}}\left\{1+\frac{c_{S \mathrm{i}}^{2}}{c_{\mathrm{Ai}}^{2} c_{\mathrm{i}}^{2}}\left[R_{L} R_{\rho} \frac{c_{\mathrm{Ai}}^{4}}{c_{\mathrm{i}}^{2}}+c_{\mathrm{Ae}}^{2}\right.\right. \\
& \left.\left.-\left(v_{0 \mathrm{i}} \pm c_{T \mathrm{i}}-v_{0 \mathrm{e}}\right)^{2}\right] \frac{\theta^{2}}{8 \sin ^{2} \frac{\Phi}{2} R_{L} R_{\rho}}\right\}
\end{aligned}
$$

and

$$
\begin{gathered}
c \approx v_{0 \mathrm{e}} \pm c_{T \mathrm{e}}\left\{1+\frac{c_{S \mathrm{e}}^{2}}{c_{\mathrm{Ae}}^{2} c_{\mathrm{e}}^{2}}\left[\frac{c_{\mathrm{Ae}}^{4}}{c_{\mathrm{e}}^{2}} \frac{1}{R_{L} R_{\rho}}+c_{\mathrm{Ai}}^{2}\right.\right. \\
\left.\left.-\left(v_{0 \mathrm{e}} \pm c_{T \mathrm{e}}-v_{0 i}\right)^{2}\right] \frac{\theta^{2} R_{\rho}}{8 \sin ^{2} \frac{\Phi}{2} R_{L}}\right\} .
\end{gathered}
$$


In a compressible plasma, body modes can also propagate and their dispersion relation is given by

$$
\frac{1}{2}\left(S-\frac{1}{S}\right)=\frac{\cos \Phi}{\sin \left(q_{\mathrm{i}} \theta\right) \sinh \left(\frac{q_{\mathrm{e}} \theta}{R_{L}}\right)}-\cot \left(q_{\mathrm{i}} \theta\right) \operatorname{coth}\left(\frac{q_{\mathrm{e}} \theta}{R_{L}}\right)
$$

In the slender slab approximation $(\theta \ll 1)$ the above dispersion relation becomes

$$
-\frac{4 \sin ^{2} \frac{\Phi}{2}}{\theta^{2}} \approx-q_{\mathrm{i}}^{2}+\frac{q_{\mathrm{e}}^{2}}{R_{L}^{2}}+\left(S-\frac{1}{S}\right) \frac{q_{\mathrm{i}} q_{\mathrm{e}}}{R_{L}} .
$$

Using the same method as in the case of surface waves, we obtain that the dispersion relation of body waves in the thin slab approximation is similar to the equation describing surface modes to the second order terms in Eqs. (42), (43). Since the second terms in these equations contain a term proportional to the very small $\theta^{2}$, changing the sign from plus (surface modes) to minus (body modes) will not affect our results considerably.

At the other limit $(\theta \gg 1)$, surface waves will propagate with phase speeds given by

$S^{( \pm)}+1=0$

which means that the propagation speed of surface waves $\left(c_{\infty}\right)$ is the root of the equation

$$
R_{\rho}+c_{\mathrm{e}}^{2} c_{\mathrm{i}}^{2} \frac{\left(c_{T \mathrm{e}}^{2}-\tilde{c}_{\mathrm{e}}^{2}\right)\left(c_{T \mathrm{i}}^{2}-\tilde{c}_{\mathrm{i}}^{2}\right)}{\left(c_{S \mathrm{e}}^{2}-\tilde{c}_{\mathrm{e}}^{2}\right)\left(c_{S \mathrm{i}}^{2}-\tilde{c}_{\mathrm{i}}^{2}\right)}=0
$$

Surface waves in the wide slab approximation (or the short wavelength limit if we keep the geometrical size of the structure constant) do not contain information about the size of the waveguide nor information about the periodicity, i.e. they are not dispersive. Equation (46) also implies that the quantity $S^{( \pm)}$ must be negative, i.e. according to Eq. (12) the phase speed of surface waves in the wide slab limit must be between the two Doppler-shifted Alfvén speeds.

In the wide-slab limit, body waves will be governed by the equation

$\cot \left(q_{\mathrm{i}} \theta\right)+\frac{1}{2}\left(S^{( \pm)}-\frac{1}{S^{( \pm)}}\right)=0$

Compared to the behaviour of surface waves, the dispersion relation of body waves still contains information about the size of the waveguide, i.e. they are dispersive.

Now, we apply these theoretical results to study the propagation of linear compressional waves in various solar structures which can be considered as periodic (or quasi-periodic) waveguides.

\subsection{Waves in photospheric structures}

A possible photospheric application would be the study of wave propagation in sunspot penumbrae. Seen in white light, a sunspot consists of the dark, central umbra surrounded by the brighter, radially striated penumbra made of alternating bright and dark filaments with diameters around $100 \mathrm{~km}$ (Martinez Pillet 2000). If seen in $\mathrm{H}_{\alpha}$, penumbrae show a much more elongated structure (the so-called superpenumbra), sometimes as much as twice the sunspot's radius in white light. Penumbrae can be also seen in X-ray, at coronal temperatures when the filamentary features emanate from the inner penumbra and extend much further outward, to distances comparable to the solar radius. The magnetic field in the umbra is vertical, but close the penumbra, the field lines tend to be horizontal, relative to the solar surface. Recent observations by Langhans et al. (2005) suggest that, while the magnetic field in the dark filaments is nearly horizontal, the field in the bright filaments is inclined to the horizontal with a mean inclination of $10^{\circ}-15^{\circ}$. However, here we will suppose that in both types of filaments, the magnetic field is horizontal, with the magnetic field strength being somewhat weaker in dark regions than in the bright ones.

Observations also show that there is a systematic inward (towards the umbra) plasma flow in the bright elements of the umbra and outward Evershed flow occurring mostly in the dark filaments (see, e.g. Borrero et al. 2005). The most important motion in the penumbra is the radial, nearly horizontal (field aligned) outflow of plasma responsible for the Evershed effect seen in spectral lines formed in the penumbral photosphere. This motion is thought to be a siphon flow driven by a pressure difference between the footpoints of arched magnetic flux tubes. The siphon flow is probably also responsible for the reversed, inward Evershed flow seen higher up in the penumbral atmosphere, in chromospheric spectral lines such as $\mathrm{H}_{\alpha}$ (Schlichenmaier 2002).

Radially outward propagating running penumbral waves have speeds of $10-20 \mathrm{~km} \mathrm{~s}^{-1}$, repeating with a period in the range of $200-300 \mathrm{~s}$ with a horizontal wavelength of 2.3-3.8 Mm. Since these speeds are larger than the local sound speed, these waves might be fast magnetoacoustic waves. Penumbral structures also support waves with periods around 5 min, which are related to the interaction of the sunspot with the resonant acoustic ( $p$-modes) in the surrounding quiet Sun. Georgakilas et al. (2000) reported slow inward propagating waves in the photospheric penumbra and outward propagating waves in the area around the sunspot. The phase speed of these waves was near $0.5 \mathrm{~km} \mathrm{~s}^{-1}$ in both cases, and their horizontal wavelength was about $2.5 \mathrm{Mm}$.

The possible modes arising in penumbral fine structures modelled as a periodic structure are shown in Fig. 5. Dispersion curves are obtained for two values of $\Phi(0, \pi / 2)$ and for each value of $\Phi$ the dispersion relation is plotted for three values of $R_{L}(0.1,2.5,10)$. We consider the system of periodic structures in such a way that the bright features are described by the field $B_{\mathrm{i}}$ and the dark ones by $B_{\mathrm{e}}$ and $B_{\mathrm{i}} / B_{\mathrm{e}}>1$. We normalize all characteristic speeds to the internal Alfvén speed and in addition we use $a_{S \mathrm{i}}=c_{S \mathrm{i}} / c_{\mathrm{Ai}}, a_{S \mathrm{e}}=c_{S \mathrm{e}} / c_{\mathrm{Ai}}$ with

$a_{T \mathrm{i}}=\frac{c_{T \mathrm{i}}}{c_{\mathrm{Ai}}}, \quad a_{T \mathrm{e}}=\frac{c_{T \mathrm{e}}}{c_{\mathrm{Ai}}}$.

With an Alfvén speed in bright regions of $9 \mathrm{~km} \mathrm{~s}^{-1}$, we choose $c_{S \mathrm{i}}=12 \mathrm{~km} \mathrm{~s}^{-1}$ and $c_{S \mathrm{e}}=11 \mathrm{~km} \mathrm{~s}^{-1}$ with $v_{\mathrm{Ae}}=6 \mathrm{~km} \mathrm{~s}^{-1}$. For the equilibrium flow we choose $M_{\mathrm{i}}=-0.1$ and $M_{\mathrm{e}}=0.2$. The fact that the bright regions are somewhat hotter than the dark ones is in agreement to the results by Borrero et al. (2005), 


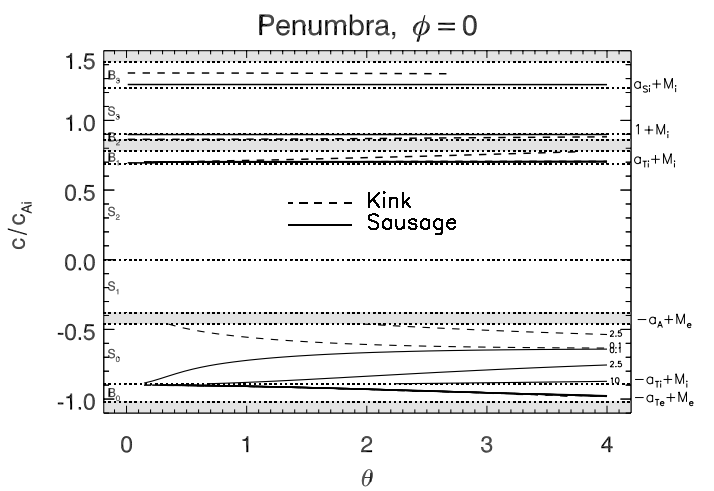

Fig. 5. The phase speed of sausage (solid line) and kink modes (dashed lines) as a function of the dimensionless quantity, $\theta$, arising in a compressible periodic plasma modelling waves in the penumbra for $\Phi=0$. Speeds are expressed in internal Alfvén speed units, $S$ and $B$ denote the speed intervals where surface and body modes are allowed.

and this difference is true over $70 \%$ of the penumbral structure. After this point, the bright regions become cooler.

For these characteristic speeds, slow surface propagating waves are allowed to propagate with phase speeds less than the Doppler-shifted internal cusp speed while fast surface waves can propagate if their phase speed is between the Doppler-shifted internal Alfvén speed and sound speed. Body waves propagate in such away that slow body modes exist between the Doppler-shifted internal and external cusp speeds, fast body modes between the shifted external and internal Alfvén speeds and in between the Doppler shifted internal and external sound speeds. A completely new behavior appears for waves propagating in the opposite direction (negative phase speeds), towards the umbra. Due to the existing background steady flow, the propagating limits are mixed so that now surface waves will propagate between $\left[-c_{T \mathrm{i}}+v_{0 \mathrm{i}}\right]$ and $\left[-v_{\mathrm{Ae}}+v_{0 \mathrm{e}}\right]$, while slow body modes will propagate for phase speeds within the interval $\left[-c_{S \mathrm{e}}+v_{0 \mathrm{e}},-c_{T \mathrm{i}}+v_{0 \mathrm{i}}\right]$, with backward propagating slow waves moving faster than backward fast waves. From Figs. 5, 6 we notice the absence of forward propagating surface waves. The absence of these modes can be partially confirmed by analyzing the dispersion relation for surface waves in the long and short wavelength limit. For the given propagation limits, the dispersion relations do not have solutions. In contrast, surface waves can propagate in the backward direction, i.e. towards the sunspot. We have omitted labelling the curves with the corresponding $R_{L}$ for modes which are practically independent of this quantity. For all values of $\Phi$, forward propagating body waves are very weakly dispersive, waves propagating in the opposite direction have a stronger dispersion, surface waves being the most dispersive modes. For all values of $\Phi$, all body modes show a dispersion relation almost independent of the ratio $R_{L}$, while for surface waves we see a clear difference in behavior for different values of $R_{L}$. This can be understood bearing in mind that the dominant dynamics of body modes occurs inside the waveguide, while for surface waves this occurs at the boundary. The dispersion diagram obtained for $\Phi=\pi$ resembles very much the diagram obtained for $\Phi=\pi / 2$; only a small shift towards smaller wavelengths (or larger $\theta$ ) is

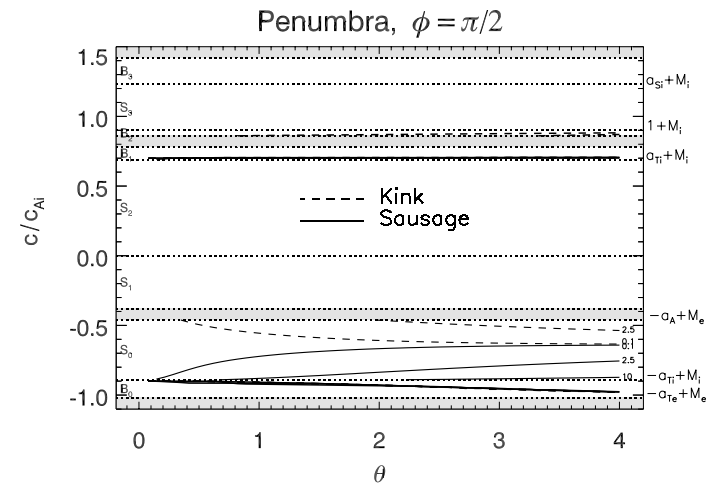

Fig. 6. The same as in Fig. 5 but $\Phi=\pi / 2$.

observed. With increasing $\Phi$, we observe the disappearance of fast body modes propagating with phase speeds larger than the Doppler shifted internal sound speed, modes which were present for $\Phi=0$. For $\Phi=\pi / 2$, only fast kink modes are observed appearing for values of $\theta$ close to unity. Obviously, the model we presented here is a simplified approximation of the complicated penumbra structure. The appearance/disapparance of modes is greatly influenced by the flow strengths and the direction of the plasma flows. A small change in the internal flow speed would allow surface waves to propagate outward.

\subsection{Waves in coronal and solar wind structures}

Plumes are an intrinsic feature of polar coronal holes. They appear in white-light coronagraphs and at eclipse as ray-like structures, extending up to many solar radii above the solar limb. The study of coronal plumes became an important issue of modern solar physics after they were observed in extreme ultraviolet (EUV) by SOHO and TRACE. While plumes may well not contribute significantly to the fast solar wind, they are major features of coronal holes. Plumes (together with their surroundings, the interplume region) require detailed theoretical modelling. They occur in magnetic field concentrations situated at the corners of the network cells.

Observational evidence of compressional waves in the solar wind has only been obtained recently. DeForest \& Gurman (1998), using high-cadence EIT/SOHO observations, found quasi periodic fluctuations with periods of $10-15 \mathrm{~min}$ in solar plumes, with a filamentary structure within the plume on a spatial scale of 3-5 arcsec. These fluctuations were identified as either sound or slow magnetoacoustic waves propagating along the plumes at $75-150 \mathrm{~km} \mathrm{~s}^{-1}$. Ofman et al. (2000) and Banerjee et al. (2001) detected quasi periodic variations (identified as slow waves) in the polarization brightness at $1.9 R_{\odot}$ in both plume and interplume regions. Their Fourier power spectrum shows significant peaks at around 1.6-2.5 mHz. In order to study the possible modes in periodic structures in the coronal holes modelling the plume/interplume region, we choose characteristic speeds of $v_{\mathrm{Ai}}=750 \mathrm{~km} \mathrm{~s}^{-1}, c_{S \mathrm{i}}=140 \mathrm{~km} \mathrm{~s}^{-1}, v_{\mathrm{Ae}}=$ $1350 \mathrm{~km} \mathrm{~s}^{-1}, c_{S \mathrm{e}}=120 \mathrm{~km} \mathrm{~s}^{-1}$, thus the plume/interplume density ratio is 3.2 . According to the line-width measurements in the plume/interplume region we choose an equilibrium flow of $65 \mathrm{~km} \mathrm{~s}^{-1}$ in the plume and $40 \mathrm{~km} \mathrm{~s}^{-1}$ in the interplume 


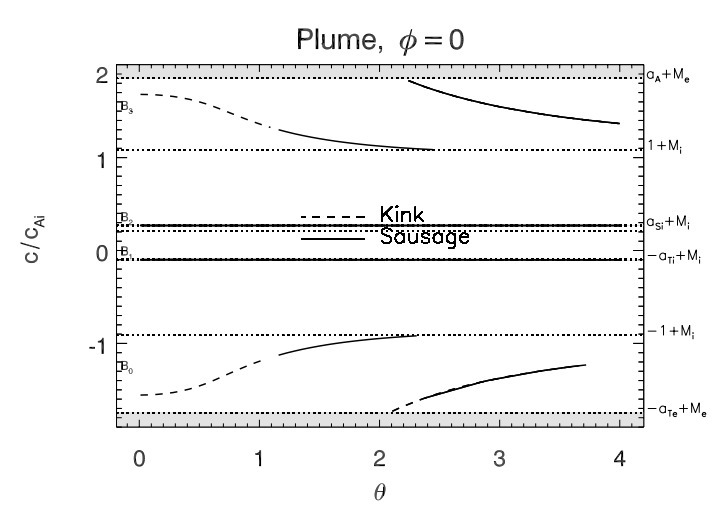

Fig. 7. The phase speed of sausage (solid lines) and kink modes (dashed lines) as a function of the dimensionless quantity, $\theta$, arising in a compressible periodic plasma modelling waves in coronal plumes for $\Phi=0$. Both forward and backward modes have distinctive dispersion curves only for $R_{L}=0.1$, for larger values the dispersion curves are very close to each other.

region (Wilhelm et al. 2000). Although all flows seem to originate from the Sun, an inflow in the plume cannot be ruled out (Raju et al. 2000). However, in the present study we will focus only on outwardly flowing cases.

The possible modes propagating in a plume are shown in Figs. 7 and 8. Similarly to the results obtained previously for the coronal case (see, e.g. Edwin \& Roberts 1983), plumes support the propagation of body waves only. The dispersion relations of linear compressional modes are plotted with respect to the dimensionless quantity $\theta$ for forward and backward propagation for $\Phi=0$ and $\Phi=\pi / 2$ and for three values of $R_{L}$ $(0.1,2.5,10)$. Since the Mach numbers are small, the effect of the flow is expected to be small. When $\Phi=0$ (Fig. 7) slow body waves (forward and backward) are very weakly dispersive and they propagate with phase speeds close to the tube speed. Fast waves are strongly dispersive. As we can see, only kink modes are able to propagate for long wavelengths (i.e. wavelengths larger than the radius of the waveguide) and fast kink modes do not have a cut-off for $R_{L}=0.1$. When $\theta \approx 1$ the kink modes are replaced by sausage modes for which the propagation speed decreases with decreasing wavelength. The first modes appear for $R_{L}=0.1$; for larger values of $R_{L}$ the dispersion curves are shifted towards smaller wavelengths and the modes are practically independent of $R_{L}$. For $\Phi=\pi / 2$ (Fig. 8) slow body modes are again very weakly dispersive, and changes are observed only in the case of fast body waves. For long wavelengths ( $\operatorname{small} \theta$ ) only kink modes propagate for $R_{L}=0.1$ but no modes are observed for $\theta \ll 1$. Compared to the case when $\Phi=0$ now even the mode corresponding to $R_{L}=0.1$ has a cutoff. Comparing the dispersion curves in the last two figures, it is obvious that the dependence of the modes on the quantity $R_{L}$ becomes more distinctive with increasing $\Phi$; dispersion curves migrate towards larger wavelengths for increasing $R_{L}$. For $\Phi=\pi$ the dispersion curves are similar to the curves obtained in the case $\Phi=\pi / 2$, the only observed difference is the displacement of the modes towards smaller wavelengths with no modes in the long wavelength limit.

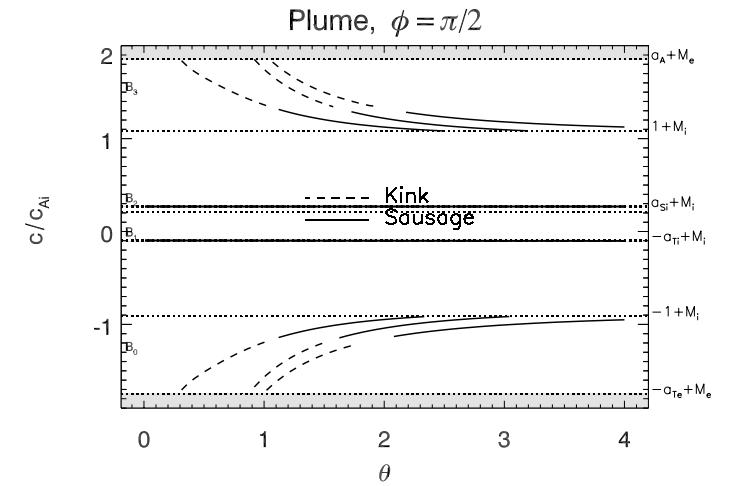

Fig. 8. The same as in Fig. 7 but now $\Phi=\pi / 2$. Both forward and backward modes have distinctive dispersion curves; for larger values of $R_{L}$ the dispersion curves are moving towards smaller wavelengths.

Further out in the solar wind, the plasma forms the socalled "spaghetti structures". Recent observations made by the HELIOS spacecraft have revealed fine structures in high-speed solar wind flows. These structures are in the form of thin flow layers (or tubes) that are adjacent to each other with differences in their plasma parameters (density, magnetic field, steady flow-speed, see e.g. Thieme et al. 1990). The structures can be separated by tangential discontinuities in the magnetic field, across which the total (kinetic plus magnetic) pressure is continuous. Parker (1963) was the first to predict the existence of such long and thin spaghetti-like stream structures as constituent units of the solar wind high-speed streams. These structures originate in the Sun, from where they spread out in the heliosphere, thus maintaining their identity even at a distance of $1 \mathrm{AU}$ from the Sun. For solar wind spaghetti structures, we choose $v_{\mathrm{Ai}}=75 \mathrm{~km} \mathrm{~s}^{-1}, c_{S \mathrm{i}}=65 \mathrm{~km} \mathrm{~s}^{-1}$, $v_{\mathrm{Ae}}=100 \mathrm{~km} \mathrm{~s}^{-1}$ and $c_{S \mathrm{e}}=70 \mathrm{~km} \mathrm{~s}^{-1}$, which means that $R_{\rho}=1.48$ (Joarder et al. 1997; Miteva et al. 2004). For the flow, we choose $v_{0 \mathrm{i}}=500 \mathrm{~km} \mathrm{~s}^{-1}\left(M_{\mathrm{i}}=6.66\right)$ and $v_{0 \mathrm{e}}=450 \mathrm{~km} \mathrm{~s}^{-1}$ $\left(M_{\mathrm{e}}=6\right)$. The possible modes arising in spaghetti structures for $\Phi=0$ and $\Phi=\pi / 2$ are shown in Figs. 9 and 10. First of all, spaghetti structures do not allow surface waves, a result pointed out earlier by Nakariakov et al. (1996). The periodicity in the model does not introduce new types of waves, the absence of surface waves because $\beta<1$ is valid for both configurations (single and periodic slabs). Forward and backward propagating waves are allowed, but due to the high Mach number the backward propagating waves are advected by the outwardly flowing plasma (away from the Sun), so they will propagate away from the Sun. Inspecting Fig. 9, we can see that only slow body modes (sausage and kink) can propagate forward and they are very weakly dispersive, the $R_{L}$ ratio does not influence significantly the propagation speed of modes. The dispersion diagram also shows slow and fast backward propagating waves, with slow waves actually propagating faster than fast magnetoacoustic modes. Slow modes show little variation with respect to the dimensionless quantity $\theta$. Fast waves have stronger dispersion and there is a clear dependence on $R_{L}$. Fast sausage and kink modes for which $R_{L}=0.1$ have their propagation speed increasing considerably with $\theta$, the other fast waves have little dispersive variation. When $\Phi=\pi / 2$ forward and backward slow waves have again small dispersive character, 


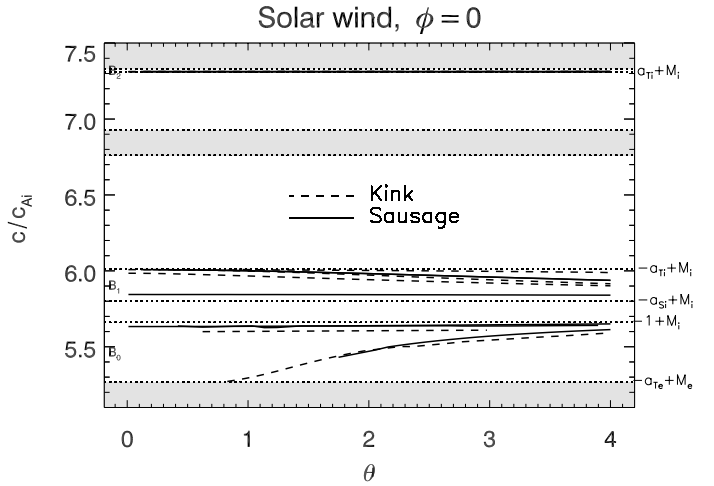

Fig. 9. The phase speeds of sausage (solid line) and kink modes (dashed lines) as functions of the dimensionless quantity, $\theta$, arising in a compressible periodic plasma modelling the solar wind spaghetti structures for $\Phi=0$.

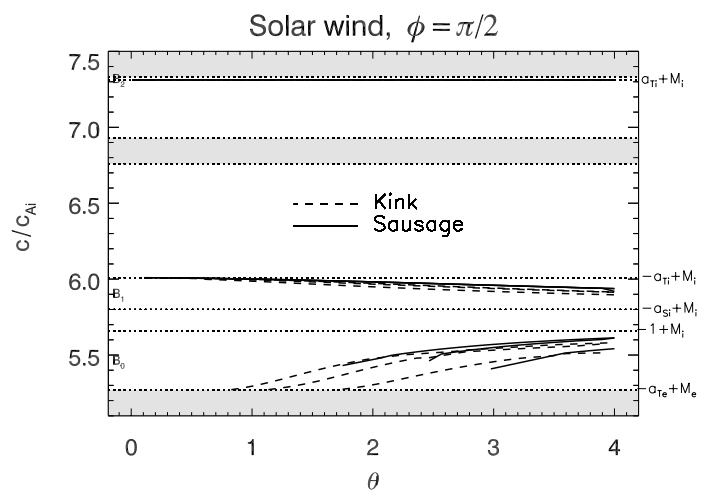

Fig. 10. The same as in Fig. 9 but now $\Phi=\pi / 2$. Only backward fast modes have distinctive dispersion curve; for larger values of $R_{L}$ the dispersion curves are moving towards smaller wavelengths.

while backward fast sausage and kink modes are strongly dispersive. As $R_{L}$ increases the dispersive curves move towards larger values of $k$ (smaller wavelengths). Fast kink modes have a cut-off value at $\left[-c_{T \mathrm{e}}+v_{0 \mathrm{e}}\right]$. Sausage modes appear only for $\theta>1$. When $\Phi$ is increased to $\pi$, the displacement of backward fast sausage and kink modes towards larger values of $k$ is more pronounced and the dispersion curves of forward and backward slow waves are similar to the case found when $\Phi=\pi / 2$.

An illustrative case on the effects of the periodicity on the propagation of waves in solar wind spaghetti structures can be found by comparing the results obtained by Nakariakov et al. (1996) with the results obtained in the present model. We suppose the same characteristic speeds as in the paper by Nakariakov et al. (1996) $\left(a_{\mathrm{A}}=1.54, a_{\mathrm{Se}}=1.08, a_{T \mathrm{e}}=0.88\right.$, $M=1.46$ and now the flow is the relative flow). For this particular case, the authors found no forward waves, only backward modes were able to propagate away from the Sun. The dispersion curves found by Nakariakov et al. (1996, Fig. 3 in their paper) show only backward fast kink modes, and backward slow body sausage and kink modes. We have repeated the same analysis but now considering a periodic spaghetti structure. The dispersion curves are shown in Fig. 11 for $\Phi=0$ and Fig. 12 for $\Phi=\pi / 2$. By considering a periodic geometrical equilibrium slow modes have the same behaviour but now they are forward

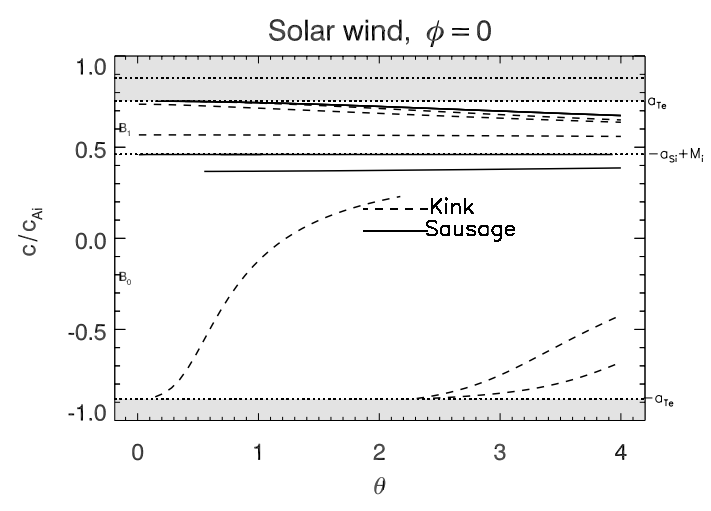

Fig. 11. The phase speed of sausage (solid line) and kink modes (dashed lines) as a function of the dimensionless wavelength, $\theta$, arising in a compressible periodic plasma modelling waves in solar wind spaghetti structures for $\Phi=0$. The dispersion curves are obtained for the same equilibrium values as Nakariakov et al. (1996). Only backward fast kink modes have distinctive dispersion curves; for larger values of $R_{L}$ the dispersion curves are moving towards smaller wavelengths.

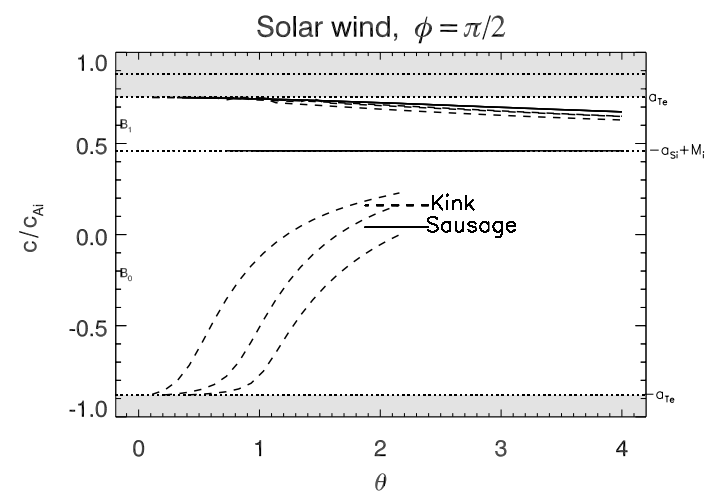

Fig. 12. The same as in Fig. 11 but now $\Phi=\pi / 2$. Only backward fast modes have distinctive dispersion curve; for larger values of $R_{L}$ the dispersion curves are moving towards larger wavelengths.

slow sausage and kink modes. Nakariakov et al. (1996) found only backward propagating fast kink modes. Inspecting the interval between $\left[-a_{T \mathrm{e}},-a_{S \mathrm{i}}+M\right]$ we can observe backward fast sausage and kink modes. Sausage waves propagate with phase speeds very close to the Doppler shifted sound speed and they are very weakly dispersive. For kink modes the situation is very different from the results obtained by Nakariakov et al. (1996). While they did not find modes propagating towards the Sun, in the case of periodic structures, we can observe that for $R_{L}=0.1$ (the first mode from left) the fast kink mode propagates towards the Sun until $\theta=1.2$, after which the wave will propagate away from the Sun despite being still backward. Increasing $R_{L}$ (the last two modes) modes will propagate towards the Sun and they appear only for $\theta>2.3$. In fact, the dispersion diagram in Nakariakov et al. (1996) recovers only the positive part of the mode corresponding to $R_{L}=0.1$. For $\Phi=\pi / 2$ (Fig. 12) the modes corresponding to $R_{L}=2.5$ and $R_{L}=10$ are approaching to the modes we obtained for $R_{L}=0.1$ and now all three modes have positive parts. Moreover, in the diagram presented by Nakariakov et al. (1996) the fast backward modes with phase speed close to $-a_{S \mathrm{i}}+M_{\mathrm{i}}$ are missing, i.e. 
considering periodic structures means a richer spectrum of possible waves.

\section{Conclusions}

Recent high resolution ground and space based telescopes have revealed that many solar features have substructures; some are periodic or quasi-periodic in the transversal direction. Here the propagation of waves in a waveguide is likely to be influenced by the properties and the width of the neighboring structures.

We studied the propagation of linear compressional MHD waves in a periodically structured magnetic medium. Dispersion relations were derived and analysed in the limiting cases of thin and thick slabs. Theoretical results were applied to study particular cases such as waves in penumbral striations in the solar photosphere, plumes/interplumes in coronal holes and the spaghetti structures in the solar wind.

A key ingredient in our analysis was the field-aligned equilibrium steady flow present in both internal and external regions. For characteristic speeds and equilibrium flows, we have studied first the propagation of slow and fast magnetoacoustic waves in penumbrae. For this structure we obtained that surface waves propagate only towards the umbra (backward propagating waves). Forward propagating body waves are weakly dispersive and they are practically independent of the width ratio of adjacent slabs, $R_{L}$. We showed that backward propagating slow magnetoacoustic waves propagate faster than fast magnetoacoustic waves due to the presence of flows. The obtained propagating modes are very sensitive to the strength and direction of the flow.

In coronal holes, the plume/interplume system was modelled by periodically distributed slabs. Similarly to previous studies of waves under coronal conditions, only body waves can propagate. While slow waves showed very little dispersion, fast forward/backward propagating waves were dispersive Dispersion curves moved towards smaller wavelengths with increasing $R_{L}$. In the solar wind, we studied waves in spaghetti structures and we obtained that only body modes are allowed to propagate. Due to the particular values of the characteristic speeds we obtained that only slow body modes propagate in the forward direction, there is a much richer spectrum of modes propagating in the backward direction. Due to the very high Mach numbers, even the backward propagating modes are travelling away from the Sun with backward slow waves propagating faster than the fast magnetoacoustic modes. We have compared the predictions of our model with previous studies on waves in spaghetti structures and we obtained remarkable discrepancies, demonstrating that the consideration of periodical structures has an important effect on wave propagation.

The dispersion curves presented in the present study were obtained for particular values of $\Phi$. Due to the periodicity with no boundaries in the $x$-direction, $\Phi$ could take any value. If we plotted the dispersion curves for all possible values of $\Phi$, we would obtain continuous spectra, a result presented recently in the context of periodically distributed prominence fibrils by Díaz \& Roberts (2006). The problem of stability and the influence of modes on the possible modes in the neighboring slabs will be addressed in a forthcoming paper.
Acknowledgements. A.M. would like to acknowledge the financial support of the Royal Society and the warm hospitality of Dept. of Applied Mathematics (University of Sheffield) where part of this research was carried out. I.B. acknowledges the financial support by Nuffield Foundation (NUF-NAL 04) and NFS Hungary (OTKA, TO43741).

\section{References}

Athay, R. G., \& Dere, D. P. 1991, ApJ, 381, 323

Ballai, I., \& Marcu, A. 2004, A\&A, 415, 691

Banerjee, D., O'Shea, E., Doyle, J. G., \& Goossens, M. 2001, A\&A, 377, 691

Bellot Rubio, L. R., Balthasar, H., Collados, M., \& Schlichenmaier, R. 2003, A\&A, 403, 47

Berton, R., \& Heyvaerts, J. 1987, Sol. Phys., 109, 201 (BH87)

Borrero, J. M., Lagg, A., Solanki, S. K., \& Collados, M. 2005, A\&A, 436, 333

Buchlin, E., \& Hassler, D. M. 2000, in A\&AS/Solar Physics Division Meeting, 32, 201

Carter, B. K. 2005, in Plasma- and Astrophysics: from laboratory to outer space, ed. Ballai et al., PADEU, 15, 175

DeForest, C. E., \& Gurman, J. B. 1998, AJ, 501, L217

De Pontieu, B., Erdélyi, R., \& James, S. P. 2004, Nature, 430, 536

Díaz, A. J., \& Roberts, B. 2006, A\&A, submitted

Edwin, P., \& Roberts, B. 1982, Sol. Phys., 76, 239

Edwin, P., \& Roberts, B. 1983, Sol. Phys., 88, 179

Edwin, P., Roberts, B., \& Hughes, W. S. 1986, Geophys. Res. Lett., 13,373

Gabriel, A. H., Bely-Dubau, F., \& Lemaire, P. 2003, ApJ, 589, 623

Georgakilas, A. A., Christopoulou, E. B., \& Koutchmy, S. 2000, A\&A, 363, 306

Gordon, B. E., \& Hollweg, J. V. 1983, ApJ, 266, 373

Heyvaerts, J., \& Priest, E. R. 1983, A\&A, 117, 220

Hollweg, J. V. 1982, J. Geophys. Res., 87, 8065

Hollweg, J. V., \& Roberts, B. 1984, J. Geophys. Res., 89, 9703

Homem, M., Erdélyi, R., \& Ballai, I. 2003, Sol. Phys., 217, 199

James, S. P., \& Erdélyi, R. 2002, A\&A, 393, L11

Joarder, P. S., Nakariakov, V. M., \& Roberts, B. 1997, Sol. Phys., 176, 285

Krijger, J. M., Roudier, T., \& Rientord, M. 2002, A\&A, 387, 672

Langhans, K., Scharmer, G. B., Kiseman, D., et al. 2005, A\&A, 436, 1087

Mariani, F., Bavassano, B., \& Villante, U. 1983, Sol. Phys., 83, 349

Martinez Pillet, V. 2000, A\&A, 361, 734

Miteva, R., Zhelyazkov, I., \& Erdélyi, R. 2004, New J. Phys., 6, 14

Nakariakov, V. M., \& Roberts, B. 1995, Sol. Phys., 159, 213

Nakariakov, V. M., Roberts, B., \& Mann, G. 1996, A\&A, 311, 311

Ofman, L., Romali, M., Polleto, G., Noci, G., \& Kohl, J. L. 2000, ApJ, 529,592

Parker, E. N. 1963, Interplanetary Dynamical Processes (New York: Interscience)

Raju, K. P., Sakurai, T., Ichimoto, K., et al. 2000, ApJ, 543, 1044

Roberts, B. 1979, Sol. Phys., 61, 23

Roberts, B. 1981, Sol. Phys., 69, 27

Ruderman, M. S., Roberts, B., Pelinovsky, E. N., \& Petrukhin, N. S. 2001, Phys. Plasmas, 8, 2628

Schlichenmaier, R. 2002, Astron. Nachr., 323, 303

Thieme, K. M., Marsch, E., \& Schwenn, R. 1990, Ann. Geophys., 8, 713

Uralov, A. M. 2003, Sol. Phys., 218, 17

Wilhelm, K., Marsch, E., Dwivedi, B. N., et al. 1998, ApJ, 500, 1023

Winebarger, A. R., Warren, H., van Ballegooijen, A., DeLuca, E. E., \& Golub, L. 2002, ApJ, 567, L89 